\title{
A Shared Resource between Declarative Memory and Motor Memory
}

\author{
Aysha Keisler and Reza Shadmehr \\ Department of Biomedical Engineering, Johns Hopkins School of Medicine, Baltimore, Maryland 21205
}

The neural systems that support motor adaptation in humans are thought to be distinct from those that support the declarative system. Yet, during motor adaptation changes in motor commands are supported by a fast adaptive process that has important properties (rapid learning, fast decay) that are usually associated with the declarative system. The fast process can be contrasted to a slow adaptive process that also supports motor memory, but learns gradually and shows resistance to forgetting. Here we show that after people stop performing a motor task, the fast motor memory can be disrupted by a task that engages declarative memory, but the slow motor memory is immune from this interference. Furthermore, we find that the fast/declarative component plays a major role in the consolidation of the slow motor memory. Because of the competitive nature of declarative and nondeclarative memory during consolidation, impairment of the fast/declarative component leads to improvements in the slow/nondeclarative component. Therefore, the fast process that supports formation of motor memory is not only neurally distinct from the slow process, but it shares critical resources with the declarative memory system.

\section{Introduction}

The motor system maintains the ability to perform accurate movements because of an adaptive system that learns from sensory prediction errors (Wallman and Fuchs, 1998; Noto and Robinson, 2001; Mazzoni and Krakauer, 2006; Tseng et al., 2007). Adaptation exhibits multiple timescales: during a typical experiment in which a perturbation is imposed on saccades (Ethier et al., 2008) or reaching movements (Smith et al., 2006), changes in motor output are driven by a fast process that learns strongly from error but produces a motor memory that decays quickly, and a slow process that learns weakly from error but produces a motor memory that shows little decay. The idea that motor adaptation is supported by a fast and a slow process accounts for a number of phenomena, including the well studied phenomenon of rapid initial improvement in performance, followed by slow, gradual improvements (Redding and Wallace, 1996; Karni et al., 1998; Della-Maggiore and McIntosh, 2005; Anguera et al., 2010), spontaneous recovery of motor memory (Chen-Harris et al., 2008), retention properties of motor memory (Criscimagna-Hemminger and Shadmehr, 2008; Joiner and Smith, 2008), and interference between motor memories (Sing and Smith, 2010). An important question is whether the neural bases of the fast and slow processes are distinct (Medina et al., 2001) or a reflection of the multiple timescales present in the synaptic plasticity of single neurons (Fusi et al., 2007; Jirenhed et al., 2007).

Received Aug. 9, 2010; revised Aug. 27, 2010; accepted Sept. 2, 2010.

This research was supported by grants from the National Institutes of Health to R.S. (NS37422) and A.K. (F32HD060369).

Correspondence should be addressed to Aysha Keisler, Johns Hopkins University School of Medicine, 720 Rutland Avenue, 416 Traylor Building, Baltimore, MD 21205. E-mail: aysha@jhmi.edu.

DOI:10.1523/JNEUROSCI.4160-10.2010

Copyright $\odot 2010$ the authors $\quad 0270-6474 / 10 / 3014817-07 \$ 15.00 / 0$
If the fast and slow processes are neurally distinct, then there should exist certain inputs that interfere with one process but not the other. Here, we focused on the fast process, as theory suggests that this process is preferentially engaged when the motor system encounters large errors. Such errors typically produce awareness of the perturbation (Malfait and Ostry, 2004; Hwang et al., 2006), and awareness can engage the declarative memory system.

Declarative memory is a memory of facts and events, and is dependent on the medial temporal cortex (Cohen and Squire, 1980; Squire, 1992). It is curious that the properties of the hypothetical fast process (rapid learning and fast forgetting) are also those of the declarative memory system. In contrast, nondeclarative memory refers to skill and habit learning, and depending on the task, engages the cerebellum, striatum, and other structures. If the fast process is dependent on the declarative system, then a declarative task might interfere with the motor memory that has been established by the fast process.

\section{Materials and Methods}

Fifty-four volunteers participated in five experiments (mean age $=26 \pm$ 4.3 years, 24 males). Experimental procedures were approved by the Johns Hopkins University School of Medicine Institutional Review Board and each participant signed a consent form.

The experiment was a standard single-target task in which subjects held the handle of a robotic arm and made point-to-point reaching movements (Shadmehr and Mussa-Ivaldi, 1994). The robot handle and the participant's hand were obscured by a horizontal screen and a cursor was projected onto the screen to indicate the handle position. Each trial began with the cursor at a center crosshair. Participants were instructed to move the cursor to a target that was placed near the body midline at 10 $\mathrm{cm}$ from the origin. Following each trial, the robot brought the hand back to the center.

On some trials, the movement was perturbed by one of two velocitydependent curl force fields, in which $\boldsymbol{f}=\mathrm{A} \dot{\boldsymbol{x}}$ (field A) or $\boldsymbol{f}=\mathrm{B} \dot{\boldsymbol{x}}$ (field $\mathrm{B}$ ), where $\mathbf{A}=\left[\begin{array}{ll}0 & 13 ;-13 \\ 0\end{array}\right] \mathrm{N} \cdot \mathrm{s} / \mathrm{m}, \mathbf{B}=-\mathbf{A}$, and $\dot{\boldsymbol{x}}$ reflects hand velocity. 
Participants were rewarded with an animated target explosion when the movement duration was $0.5 \pm 0.07 \mathrm{~s}$ and the peak movement speed was $0.20-0.55 \mathrm{~m} / \mathrm{s}$.

On null trials, participants reached to the target unperturbed. On field trials, participants reached to the target in the presence of one of the two force fields. On error-clamp trials, participants reached to the target, but the movement was constrained to a straight line to the target via a stiff channel (spring coefficient $=2500 \mathrm{~N} / \mathrm{m}$; damping coefficient $=25 \mathrm{~N} \cdot \mathrm{s} / \mathrm{m}$ ). We recorded the force produced against the channel "wall." Learning on error-clamp trials was quantified by an adaptation index (AI), or the force that subjects produced against the channel walls at peak velocity as a percentage of the ideal force (i.e., the force that the robot would produce at the same hand velocity).

Interfering tasks. We considered two kinds of tasks, one that is thought to engage declarative memory, and one that is thought to not require this memory system. These tasks were modeled after work by Brown and Robertson (2007a, 2007b). The tasks were designed to last 3 min each.

In the memory task, participants were shown 12 word pairs (i.e., "COMFORT-ATOM," "LEGEND-BLANK"). Words were chosen from the University of Western Australia Psycholinguistic database, so that all words were of comparable frequency and familiarity ratings. Each pair was shown for $3 \mathrm{~s}$. Immediately after viewing the words, participants were then shown one word from each pair and instructed to say the corresponding word aloud. The test words were shown at a fixed pace of $3 \mathrm{~s}$ per word. The study and test phases were then repeated with the same words.

In the nonmemory task, participants were shown strings of letters (i.e., "kdinedlr"). Each string was shown at a fixed pace of $3 \mathrm{~s}$ per string. The participant was instructed to say aloud the number of vowels in the string. There were 48 strings in total, shown in groups of 12 with $3 \mathrm{~s}$ pauses between groups. No feedback was given for either task.

Experiment 1. In experiment 1 (see Fig. $2 \mathrm{~A}$ ), our aim was to interfere with the motor memory that is produced by the fast process and measure the effect of this interference during the subsequent spontaneous recovery period. We considered a reaching paradigm in which a force field perturbed the hand. After a long period of training in field A, subjects were briefly exposed to field B. Subsequently, they stopped performing the motor task and were presented with a 3 min task that engaged the declarative memory system ("memory" group), a 3 min task that did not require retaining material in declarative memory ("nonmemory" group), or simply rested for 3 min ("no task" group). In theory, the long period of training in field A results in a motor memory that is mainly due to the slow process (see Fig. 1), and the brief exposure to field B results in a motor memory that is mainly due to the fast process. Because the two fields are in the opposite directions, the two memories will compete. If the fast process shares resources with the declarative memory system, then after motor training has stopped, a declarative memory task might still interfere with the memory for field B. As a result, motor output following the declarative task should actually be greater in the interfered group because the declarative task removes the influence of the competing fast process.

Eighteen individuals were randomly assigned to three groups (six participants per group). All subjects trained on a 192-trial null block in which $1 / 8$ of the trials were error clamp (pseudorandomly distributed across the block), and the remaining trials were null trials. All participants then completed 384 field A trials (1/8 error clamp) and 20 field B trials (0 error clamp). Subsequently, participants either completed the memory task (memory group) or the nonmemory task (nonmemory group), or underwent a 3 min rest (no-task group). Finally, all participants completed 192 error-clamp trials in a test block.

Experiment 2. Here, our aim was to disrupt the hypothetical fast motor memory that is produced during the initial phase of adaptation to field $\mathrm{B}$ (see Fig. $2 B$ ). To do so, we allowed a brief exposure to field $B$, then administered the interfering task. Eighteen individuals were randomly assigned to three groups. All participants first trained in a null block (identical to experiment 1), followed by 20 field B trials (no error-clamp trials were present during this brief training period). Subsequently, participants either completed the memory task (memory group) or the nonmemory task (nonmemory group), or underwent a 3 min delay (no-task group). Finally, all participants completed 150 error-clamp trials in a test block.

Experiment 3. If our hypothesis is correct, namely, that a declarative memory task interferes with the motor memory produced by the fast process, then the same task should have no effect on the memory produced by the slow process. Experiment 3 was designed to test this idea (see Fig. 3). Here, interference conditions were applied after extended training on field $\mathrm{A}$, at which point the slow process drives motor output. Twelve individuals were randomly assigned to two groups. All participants trained in a null block (identical to experiment 1), followed by 384 field A trials (1/8 error clamp). Subsequently, participants either completed the memory task (memory group) or underwent a 3 min delay (none group). Finally, all participants completed 192 error-clamp trials in a test block.

Experiment 4. To examine the interaction between the fast and slow systems over time, participants in experiment 4 underwent the same protocol as those in experiment 1, except that they completed the test block $6 \mathrm{~h}$ after the cognitive tasks (see Fig. $4 \mathrm{~A}$ ). If the results of Brown and Robertson (2007a) apply to reaching adaption, impairment of the declarative component of the task would benefit the nondeclarative component, such that a group that experiences interference performs better at a delayed test than the group that does not experience interference. Put another way, impairment of the fast memory component might benefit the consolidation process that alters the slow memory component.

Eighteen individuals were randomly assigned to three groups (six participants per group). This experiment was identical to experiment 1 , except that there was a $6 \mathrm{~h}$ delay between the end of the $3 \mathrm{~min}$ delay and the beginning of the test block.

Experiment 5. To determine whether the effects observed in Experiment 4 were due to consolidation of field A or field B, we ran a control study in which subjects only trained on field A. Twelve individuals were randomly assigned to two groups (six participants per group). This experiment was identical to experiment 3 , except that there was a $6 \mathrm{~h}$ delay between the end of the 3 min delay and the beginning of the test block.

Data analysis. Unless stated otherwise, mean metrics were compared using Kruskal-Wallis test, a nonparametric mean rank test, due to the relatively small sample size (Kruskal and Wallis, 1952). SAS PROC MIXED (SAS Institute) was used to conduct repeated-measures analyses.

\section{Results}

Consider a simple adaptation task in which a long period of adaptation is followed by brief period of deadaptation (i.e., a long period of training in $\mathrm{A}$ is followed by training in $\mathrm{B}$, as in Fig. $1 \mathrm{~A}$ ). Let us assume that there are two states that contribute to motor output: a state that changes strongly due to error, but has poor retention (the fast state), and a state that changes little due to error, but has good retention (the slow state) (Smith et al., 2006). According to this theory, the long period of adaptation results in a motor output that is primarily supported by the slow process (Fig. $1 B$ ). When the perturbation suddenly changes, the fast process responds to the large errors, competing with the slow process and returning the motor output to near baseline. If at this point movements are executed without errors (i.e., "error-clamp" trials), behavior exhibits "spontaneous recovery" (dashed black line, Fig. $1 \mathrm{~B}$ ), as the memory that was produced by the fast process decays more rapidly than the memory produced by the slow process (Smith et al., 2006; Criscimagna-Hemminger and Shadmehr, 2008; Ethier et al., 2008). This pattern of behavior affords an excellent opportunity to isolate the fast and slow components. Because performance is the net output of fast and slow memories of opposing fields, we can measure interference on the fast system alone in this critical window. If we could somehow interfere with the memory of the fast process (Fig. $1 C$ ), then only the memory of the slow process can support motor output (red line, Fig. 1C). Therefore, the theory predicts that disruption of the fast memory will alter the pattern of spontaneous recovery, re- 
A
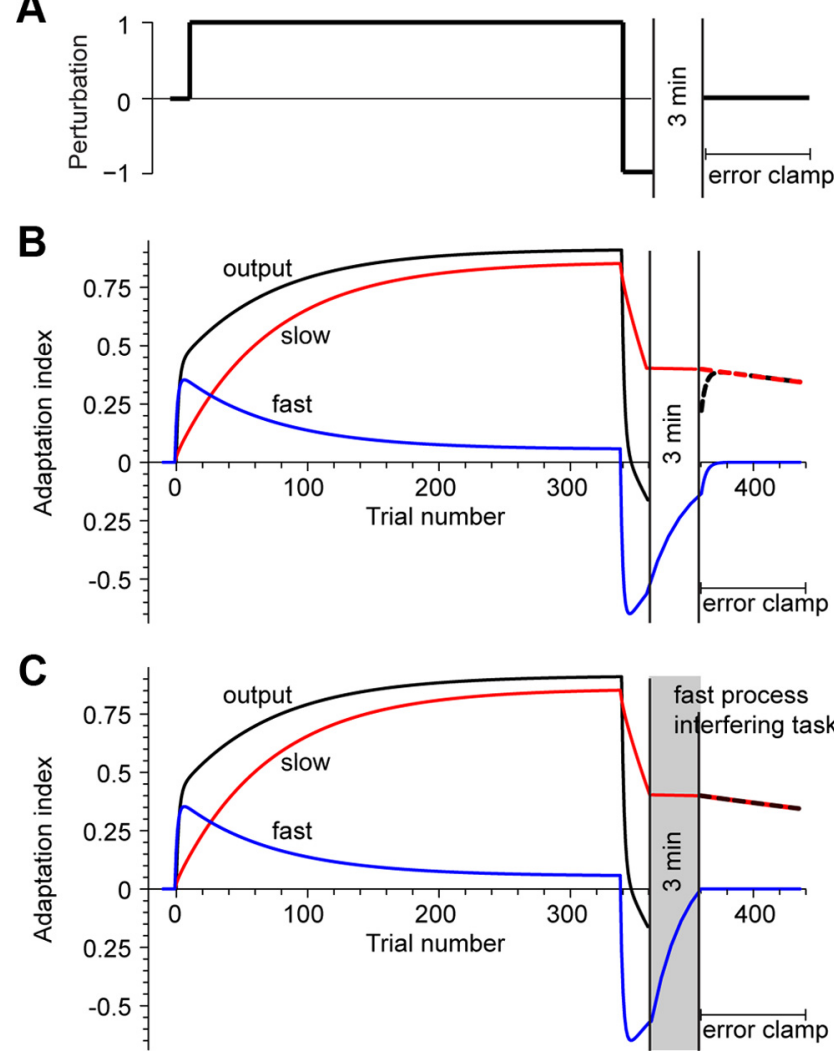

Figure 1. Model of fast and slow processes that support motor adaptation. $A$, Participants train on a null field, train for many trials in field $A$, then train briefly in field $B$ (an opposing field). After performing an interfering task, participants complete error-clamp trials in which they make movements without error. $\boldsymbol{B}$, If there is no interference, the motor output exhibits "spontaneous recovery" (dashed black line). C, Here, a declarative task occurs immediately after field $B$ training. The task may interfere with the fast component, thereby altering the patterns of spontaneous recovery.

sulting in a greater adaptive response in the positive direction (dashed black line, Fig. 1C). That is, interference with the fast memory should strengthen the initial motor output, relative to the no-interference case.

In all experiments, AIs from the null block were analyzed by creating 13 two-trial bins of the 26 error-clamp trials. Binned AIs were then subjected to repeated-measures analyses with group and bin as predictors. These predictors failed to reach significance in any of the five experiments. That is, performances in the null blocks were indistinguishable among the various groups.

In experiments 1 and 2, our aim was to interfere with the motor memory that was produced by the fast process. Our idea was that the fast process may share resources with the declarative memory system. As a result, a task that engaged the declarative memory system should alter the patterns of spontaneous recovery. For example, in experiment 1 (Fig. $2 A$ ), motor output following the declarative task should actually be greater in the interfered group because the declarative task removes the influence of the competing fast process. In experiment 1, performance was comparable among the three groups during field A, as determined by comparing mean AIs of the last eight error-clamp trials of training among the three groups, $\chi_{(2,18)}^{2}=3.03, p=0.22$. Performance was also comparable among the three groups during the subsequent field B training. We compared the mean perpendicular displacement of the last eight trials of field B training, and this analysis failed to indicate group differences, $\chi_{(2,18)}^{2}=$
0.57, $p=0.75$. We conclude that the three groups performed similarly in the A and B fields.

We next examined the error-clamp block to determine the effect of the interfering tasks on spontaneous recovery. Note that the no-task and nonmemory groups both exhibited spontaneous recovery: the forces produced in the test phase were initially low, but quickly rebounded (inset, Fig. 2A). The memory group did not show this pattern. To formally analyze these data, we conducted a repeated-measures analysis on the first 12 trials of the test block. This analysis indicated an effect of group, $F_{(2,195)}=$ $6.62, p<0.01$, and a group $\times$ trial interaction, $F_{(2,195)}=7.19, p<$ 0.01 . Further, mean rank tests showed a group effect on the first trial of the test block, $\chi_{(2,18)}^{2}=7.89, p=0.02$, but not the 12th block, $\chi_{(2,18)}^{2}=2.01, p=0.37$. Upon examination of Figure $2 A$, it can be seen that this effect is driven by the initially higher performance of the memory group, and the quick decrease in the difference between the memory group and the two control groups. We next conducted planned contrasts on this early data from the error-clamp block. The memory versus no-task contrast revealed a main effect of group, $F_{(1,195)}=11.42, p<0.01$, as did the memory versus nonmemory contrast, $F_{(1,195)}=8.16, p<0.01$. However, the nonmemory versus no-task comparison showed no difference, $F_{(1,195)}=0.27, p=0.60$. In summary, a task that engaged the declarative memory system disrupted the motor memory that was acquired earlier by the fast process, as evidenced by the altered pattern of spontaneous recovery.

In experiment 2, our aim was to disrupt the fast motor memory that is produced during the short period of adaptation to field B (Fig. 2 B). Performance of the three groups during field B training did not differ. A mean rank test of the mean maximum perpendicular displacement of the last 8 trials of training failed to show a group effect, $\chi_{(2,18)}^{2}=1.55, p=0.46$. However, as can be seen in Figure $2 B$, the performances during the subsequent errorclamp trials were quite different between the memory and the nonmemory and no-task groups. The nonmemory and no-task groups exhibited large, negative AIs in initial error-clamp trials, followed by a precipitous reduction in the magnitude of AIs in subsequent trials. The memory group, on the other hand, exhibited stable AIs of a similar magnitude as the nonmemory and no-task groups' asymptote. In other words, the motor output for the nonmemory and no-task groups starts strong then decays quickly, whereas the motor output for the memory group is poor but stable. A mean rank test on the first trial of the test block reveals a group effect, $\chi_{(2,18)}^{2}=6.45, p=0.04$. However, performance on the 12 th trial did not differ by group, $\chi_{(2,18)}^{2}=0.46, p=0.98$. Thus, the groups initially perform differently, but performance subsequently converged.

In sum, brief training on a force field task resulted in a motor memory that decayed quickly, consistent with the theory that the fast memory system supports initial learning. A posttraining declarative task interfered with this memory. This interference was specific to the declarative memory system, as an attentiondemanding cognitive task without a declarative memory component did not yield such interference.

If the declarative memory task interferes with the motor memory produced by the fast process, then the same task should have no effect on the memory produced by the slow process. In experiment 3 (Fig. 3), the groups did not differ during field A: a Wilcoxon two-sample test failed to show a group difference in the means of the last 8 trials, $Z=0.88, p=0.38$. We analyzed the error-clamp test block and as predicted saw no difference between the group that had the memory task and the control group: another Wilcoxon test on the first trial of test failed to show a 
A
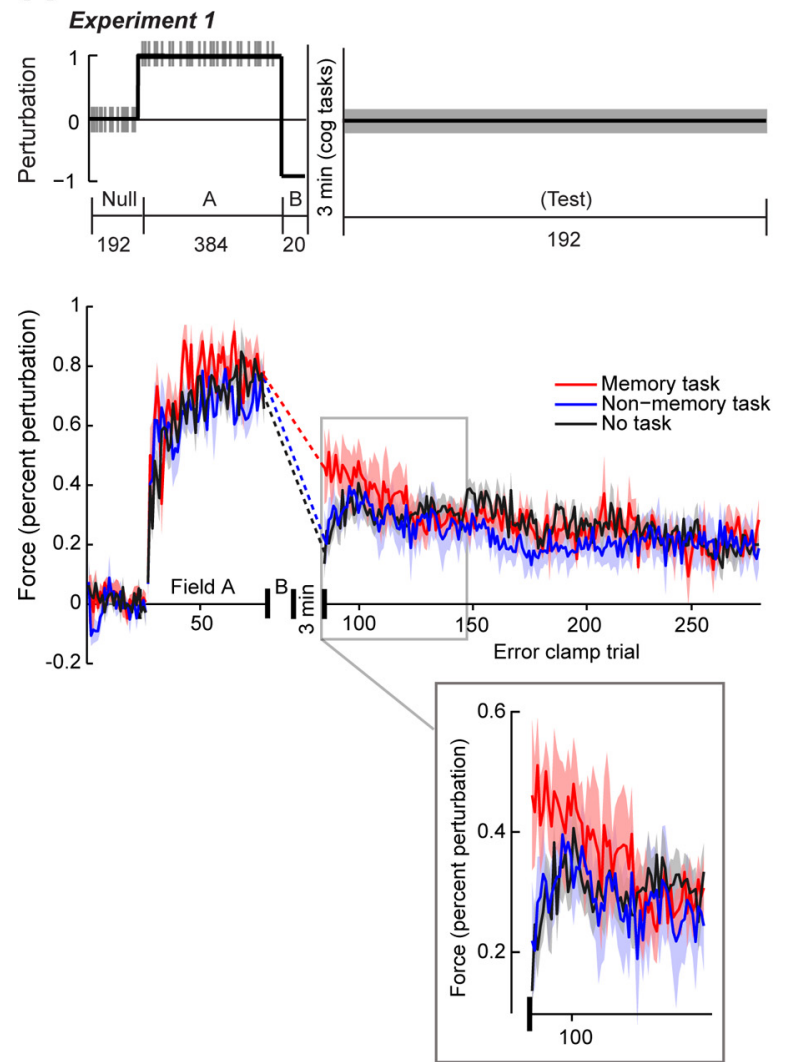

B
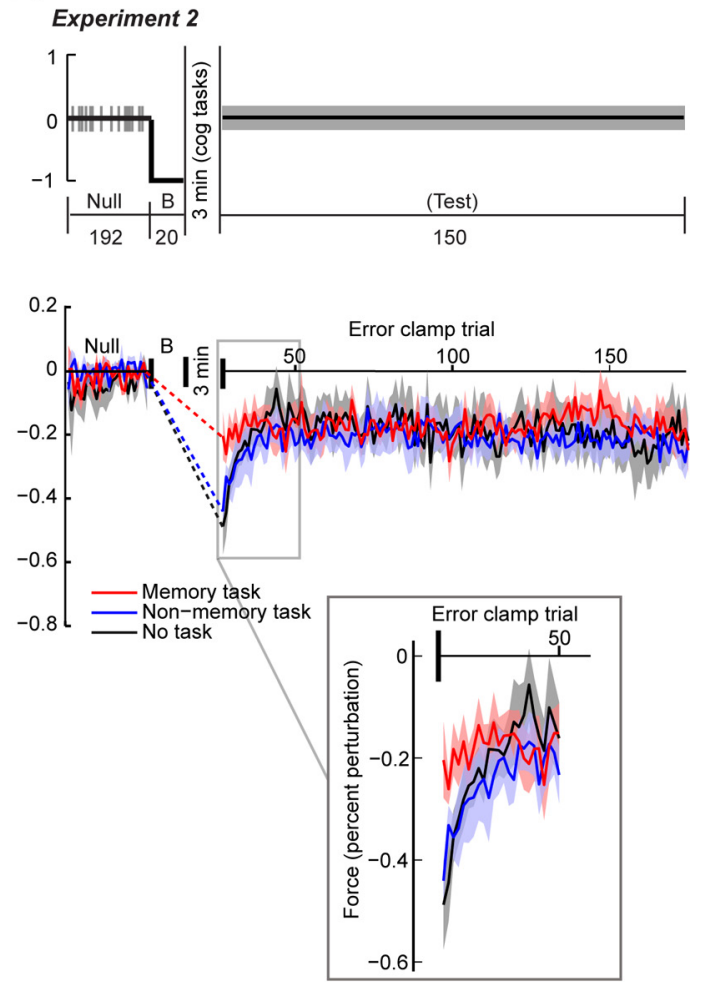

Figure 2. A, Experiment 1 experiment design (top) and performance (bottom). Participants train in a null block (no perturbation), followed by field $A$ (positive perturbation), field B (negative perturbation), and a test block (100\% error-clamp trials). Inset shows the initial trials of the test block. The "nonmemory task" and "no task" groups exhibit spontaneous recovery, but the "memory task" group does not. B, Experiment 2 design (top) and performance (bottom). Participants train in a null block (no perturbation), followed by field B (negative perturbation) and a test block (100\% error-clamp trials). The memory task disrupts the memory of field B. Vertical tick marks indicate error-clamp trials in the experiment design subfigure. Shaded regions of the data indicate SEM.

group difference, $Z=0.40, p=0.69$. In addition, a repeatedmeasures analysis on the first 12 trails revealed a main effect of trial, $F_{(1,130)}=13.35, p<0.01$, but neither an effect of group, $F_{(1,130)}=0.30, p=0.59$, nor a group $\times$ trial interaction, $F_{(1,130)}=$ $2.41, p=0.12$. Thus, the declarative memory task had no effect on performance when the motor memory was supported by the slow system.

If both declarative and nondeclarative systems are active during motor adaptation, how might the systems interact during the subsequent consolidation period? Interestingly, recent data from sequence learning literature suggests that declarative and nondeclarative systems may interact in the postacquisition period (Brown and Robertson, 2007a, 2007b). The authors suggested that impairment of the declarative component of the task improved consolidation of the nondeclarative component. To extrapolate to the adaptation framework, if the fast and slow processes are supported by declarative and nondeclarative memory systems, respectively, then impairment of the motor memory produced by the fast process should improve consolidation of the motor memory produced by the slow process. Experiment 4 (Fig. $4 A$ ) was designed to test this idea. The three groups did not differ during field A: a mean rank test on the mean AIs of the first eight trials failed to reveal a group effect, $\chi_{(2,18)}^{2}=1.30, p=0.52$. Nor did the groups differ in field B: a similar analysis on mean perpendicular displacement on the last eight trials also failed to reveal a group effect, $\chi_{(2,18)}^{2}=0,85, p=0.65$. However, a repeated-measures analysis on the first 12 trials of test reveals a main effect of group, $F_{(1,187)}=3.24, p<0.04$, indicating that AIs of memory group were lower than those of the two control groups. The trial effect and trial $\times$ group interaction failed to reach significance, $F_{(1,187)}=0.04, p=0.84$, and $F_{(1,187)}=0,17$, $p<=0.85$, respectively. That is, performance of the memory group at $6 \mathrm{~h}$ was lower than the other two groups.

There are two possible explanations for the group difference observed in experiment 4: the memory group may have (1) a greater memory for field B or (2) a weaker memory for field A, relative to the control groups. To distinguish between these possibilities, in experiment 5 (Fig. $4 B$ ) we recorded performance of participants who trained on field A only. Again, groups did not differ in training, $\chi_{(2,18)}^{2}=2.01, p=0.15$. Also, as can be seen in Figure $4 B$, performance did not differ between the memory group and the control group. This observation is borne out in repeated-measures analysis of the first 12 trials of test, which revealed a main effect of trial, $F_{(1,130)}=4.22, p=0.04$, but neither a main effect of group or a group $\times$ trial interaction, $F_{(1,130)}=$ $0.17, p=0.68$, and $F_{(1,130)}=1.40, p=0.24$, respectively. Thus, we conclude that the differences in consolidation patterns of field $\mathrm{B}$ memory accounts for the effect observed in experiment 4 . Impairment of the fast process for B benefits the memory for B after a delay.

Comparison of experiments 1 and 4 highlight an interesting dissociation: following a task that engages the declarative memory system, the memory for field B is impaired in an immediate test, but improved in a delayed test. How can the motor memory for field B strengthen after a delay, whereas it is impaired by the declarative task on an immediate test? We must assume that there 

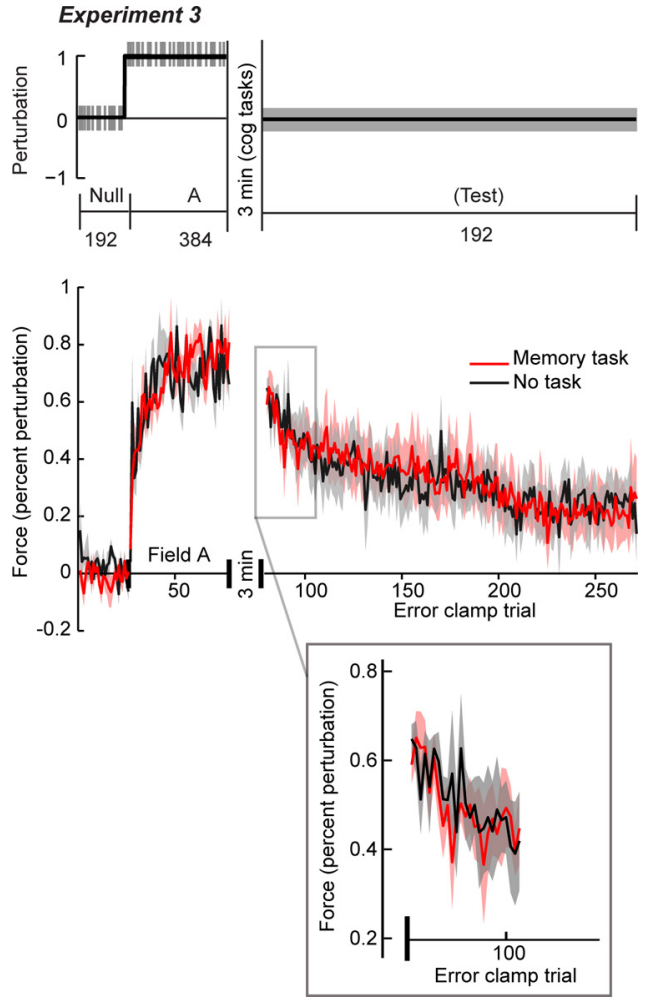

Figure 3. Experiment 3 design (top) and performance (bottom). Participants train in a null block (no perturbation), followed by field A (positive perturbation) and a test block (100\% error-clamp trials). The long period of training produced a motor memory that was not affected by the memory task. The memory task disrupts the memory of field B. Vertical tick marks indicate error-clamp trials in the experiment design subfigure. Shaded regions of the data indicate SEM.

was some degree of slow process during the 20 trials of field B training. This is clearly seen in experiment 2: even after 20 trials, the resulting memory has a component that does not decay away. In fact, Joiner and Smith (2008) demonstrated that the slow process can learn from as few as 11 trials in this type of task. If interference of the declarative system improves consolidation of the nondeclarative memory, as in the case of explicit sequence learning (Brown and Robertson, 2007a, 2007b), we would predict that interference with the fast motor memory would improve consolidation of the slow motor memory. Indeed, interference with the fast component of the memory for field B initially impedes recall for this field, but benefits consolidation of the slow component of the $\mathrm{B}$ memory. This process results in a superior slow memory for field B after a $6 \mathrm{~h}$ delay, relative to a condition in which no interference occurred.

\section{Discussion}

Behavioral changes in a variety of motor tasks, including arm movements and eye movements, suggest that acquisition of motor memory is due to a fast process that is strongly sensitive to performance errors but produces memories that decay quickly, and a slow process that is weakly affected by performance errors but produces memories that show little decay. These tasks have been typically considered examples of nondeclarative learning [e.g., amnesic individuals, including HM, learn and recall this reaching task (Shadmehr et al., 1998)]. Here, we found that the fast process shares critical resources with declarative memory: engagement of a declarative memory task after completion of the motor adaptation task produced retrograde interference with the memory produced by the fast process (experiments 1 and 2), but not the slow process (experiment 3 ). Further, neither a comparable time delay nor a control cognitive task interfered with the fast memory (experiments 1 and 2). The current data also reveal an interesting interaction between declarative and nondeclarative systems in the postacquisition consolidation process (experiments 4 and 5). Impairment of the fast/declarative component enhanced the slow/nondeclarative component after a delay, implying an inhibitory relationship between the two systems (at least, in one direction). This finding echoes similar findings in explicit sequence learning (Brown and Robertson, 2007a, 2007b). The fact that two disparate motor skills show similar effects of declarative memory on consolidation raises important questions regarding the consolidation process.

In our experiment, a declarative task had a retrograde effect on a previously acquired motor memory. Yet, the resulting framework also accounts for experiments in which declarative tasks are performed during motor adaptation. For example, we learn less from sudden, large movement errors when that error is experienced in conjunction with a competing declarative task (Taylor and Thoroughman, 2007, 2008). Similarly, we are more sensitive to trial-to-trial movement error (i.e., we learn more) under conditions in which we can verbally describe the details of the perturbation (Hwang et al., 2006). In both cases, the state of the declarative system may affect the ability of the fast process to lay down motor memories.

Our results have implications regarding the neural basis of the multiple timescales of motor memory. Cerebellar damage produces robust impairments in reach adaptation (Smith and Shadmehr, 2005; Rabe et al., 2009). There is evidence that cerebellar-dependent learning involves more than one site of plasticity, with a fast-like process associated with the cerebellar cortex and a slow-like process associated with the cerebellar nuclei (Medina et al., 2001). However, the multiple timescales of memory may also be due to the inherent timescales of plasticity within single neurons (Fusi et al., 2007), possibly in the Purkinje cells of the cerebellum (Jirenhed et al., 2007). Because we found that the fast but not the slow process shares resources with declarative memory, the multiple timescales of motor adaptation are unlikely to arise from mechanisms of plasticity within single neurons. Instead, our results are more in line with behavioral and functional imaging results that have pointed out that in the early part of training in a motor task, there are high-level strategic (Redding and Wallace, 1996), attentional (Della-Maggiore and McIntosh, 2005), and working memory (Anguera et al., 2010) processes that are not present in the later part of motor adaptation. It is possible that these prefrontal regions are a part of a cerebelloprefrontal network that forms the neuronal basis of the fast but not the slow process that generates motor memory.

The possibility that the fast and slow processes have distinct neural bases is consistent with two lines of evidence. First, training schedules that engage the fast process produce different generalization patterns than training patterns that primarily engage the slow process. For example, when force fields perturb reaching movements, sudden perturbations (engaging the fast process) produce internal models that generalize to the untrained arm (Criscimagna-Hemminger et al., 2003), whereas gradual perturbations (engaging the slow process) have no such generalization properties (Malfait and Ostry, 2004). Second, patients with severe damage to the cerebellar cortex are profoundly impaired when the perturbations are introduced suddenly, but show a latent ability to adapt if the perturbations are introduced gradually (Criscimagna-Hemminger et al., 2010). Gradual perturbations 
can lead to more robust generalization when the trained arm is used in a different context (e.g., reaching in free air after reaching with a robot), whereas this generalization is smaller if the training is in response to a sudden perturbation (Kluzik et al., 2008). Therefore, the fast process lays down a motor memory that is effector independent (i.e., one that is not tied to the body part used), and strongly depends on the integrity of the cerebellar cortex. In contrast, the slow process lays down a motor memory that is effector dependent, driven by the intrinsic coordinates of error, likely in proprioceptive coordinates. This view is consistent with evidence that early in learning, motor skills are non-effector-specific, but become increasingly effector-specific with continued training (Japikse et al., 2003; Bonzano et al., 2008). In fact, the effectordependent learning may help explain the consolidation results of experiments 4 and 5, as some theorize that motor skill consolidation is specific to the coordinate system in which learning was acquired and involves interaction between coordinate systems (Cohen and Robertson, 2007).

Perhaps the most fundamental implication of our results is that people with deficits in the declarative memory system will likely show impairments in the fast process that lays down motor memories, and will therefore show specific impairments in their ability to adapt to perturbations. This may account for the fact that whereas performance of patient HM in the force field paradigm exhibited strong retention (a measure of the consolidation of the slow process), the learning rate during acquisition was much slower than normal (a measure of acquisition due to the fast process) (Shadmehr et al., 1998).

The interaction between declarative and nondeclarative memory might also contribute to patterns of retention when the motor perturbations are introduced gradually versus suddenly. Conscious awareness of the perturbation is typically minimal when a perturbation is introduced incrementally, as opposed to instantaneously. Because awareness is a significant factor in the development of declarative memory, there may be less declarative recruitment in the incremental case that, in this framework, might account for superior retention after gradual training (Klassen et al., 2005; Huang and Shadmehr, 2009). Multiple factors, such as error size and the rate at which the perturbation is introduced, likely affect retention in tasks such as these; however, the relationship between memory systems may be one factor in posttraining consolidation.

It is surprising that a verbal task would interfere with a motor task with no overt verbal component, both in the current experiments and previous experiments on sequence learning (Brown and Robertson, 2007a, 2007b). Indeed, one would think that the declarative component here is spatial in nature, in that participants report that they learn which direction to push (this observation was not formally tested). In fact, a recent study showed that spatial skill predicts performance on a visuomotor task (Anguera et al., 2010). There is ample evidence that verbal and spatial memories are encoded in different brain regions (Shallice et al., 1994; Moscovitch et al., 1995; Heil et al., 1996); so, why does interference occur? There are at least two possible explanations. First, there may be a strong verbal component in the tasks as well as the spatial component, which is interfered with by the verbal task. This is consistent with the study by Mazzoni and Krakauer (2006), wherein manipulation of an explicit strategy (which likely has a verbal component) influenced performance. Another possibility is that while spatial and verbal memories are modular in terms of encoding, interference can occur between modalities. However, spatial and verbal working memory buffers do not ex- 
hibit such interference (Smith et al., 1996; Baddeley, 1999). Thus, it is perhaps more likely that the verbal task administered here interfered with a verbal representation of how to complete the task. In other words, the task is not "process pure" (Jacoby, 1991): though the task is primarily motoric, performance can be supported by nonmotor memory systems.

There are a number of limitations in our work. The present data only present a single dissociation, as we demonstrated that a declarative task interfered with a motor task. It is possible that the memory task interfered with the ability of the fast system to be expressed in error-clamp trials, without interfering with the memory per se. In this scenario, we must also posit some shared resource between the fast system and declarative memory in order for the memory task to affect retrieval of the fast memory, though the nature of the relationship between memory systems may be more complicated than presented here. Future work will reveal whether interference is bidirectional, and how the pattern of interference may affect consolidation.

In summary, our data show that the declarative memory system shares critical resources with the fast process of motor memory but not the slow process. Our results raise the possibility that distinct neural bases support these two processes.

\section{References}

Anguera JA, Reuter-Lorenz PA, Willingham DT, Seidler RD (2010) Contributions of spatial working memory to visuomotor learning. J Cogn Neurosci 22:1917-1930.

Baddeley AD (1999) Essentials of human memory. Hove, UK: Psychology Press.

Bonzano L, Tacchino A, Roccatagliata L, Abbruzzese G, Mancardi GL, Bove M (2008) Callosal contributions to simultaneous bimanual finger movements. J Neurosci 28:3227-3233.

Brown RM, Robertson EM (2007a) Inducing motor skill improvements with a declarative task. Nat Neurosci 10:148-149.

Brown RM, Robertson EM (2007b) Off-line processing: reciprocal interactions between declarative and procedural memories. J Neurosci 27:10468-10475

Chen-Harris H, Joiner WM, Ethier V, Zee DS, Shadmehr R (2008) Adaptive control of saccades via internal feedback. J Neurosci 28:2804-2813.

Cohen DA, Robertson EM (2007) Motor sequence consolidation: constrained by critical time windows or competing components. Exp Brain Res 177:440-446.

Cohen NJ, Squire LR (1980) Preserved learning and pattern-analyzing skill in amnesia: dissociation of knowing how and knowing that. Science 210:207-210.

Criscimagna-Hemminger SE, Shadmehr R (2008) Consolidation patterns of human motor memory. J Neurosci 28:9610-9618.

Criscimagna-Hemminger SE, Donchin O, Gazzaniga MS, Shadmehr R (2003) Learned dynamics of reaching movements generalize from dominant to nondominant arm. J Neurophysiol 89:168-176.

Criscimagna-Hemminger SE, Bastian AJ, Shadmehr R (2010) Size of error affects cerebellar contributions to motor learning. J Neurophysiol 103:2275-2284.

Della-Maggiore V, McIntosh AR (2005) Time course of changes in brain activity and functional connectivity associated with long-term adaptation to a rotational transformation. J Neurophysiol 93:2254-2262.

Ethier V, Zee DS, Shadmehr R (2008) Spontaneous recovery of motor memory during saccade adaptation. J Neurophysiol 99:2577-2583.

Fusi S, Asaad WF, Miller EK, Wang XJ (2007) A neural circuit model of flexible sensorimotor mapping: learning and forgetting on multiple timescales. Neuron 54:319-333.

Heil M, Rösler F, Hennighausen E (1996) Topographically distinct cortical activation in episodic long-term memory: the retrieval of spatial versus verbal information. Mem Cognit 24:777-795.

Huang VS, Shadmehr R (2009) Persistence of motor memories reflects statistics of the learning event. J Neurophysiol 102:931-940.

Hwang EJ, Smith MA, Shadmehr R (2006) Dissociable effects of the implicit and explicit memory systems on learning control of reaching. Exp Brain Res 173:425-437.
Jacoby LL (1991) A process dissociation framework: separating automatic from intentional uses of memory. J Mem Lang 30:513-541.

Japikse KC, Negash S, Howard JH Jr, Howard DV (2003) Intermanual transfer of procedural learning after extended practice of probabilistic sequences. Exp Brain Res 148:38-49.

Jirenhed DA, Bengtsson F, Hesslow G (2007) Acquisition, extinction, and reacquisition of a cerebellar cortical memory trace. J Neurosci 27:2493-2502.

Joiner WM, Smith MA (2008) Long-term retention explained by a model of short-term learning in the adaptive control of reaching. J Neurophysiol 100:2948-2955.

Karni A, Meyer G, Rey-Hipolito C, Jezzard P, Adams MM, Turner R, Ungerleider LG (1998) The acquisition of skilled motor performance: fast and slow experience-driven changes in primary motor cortex. Proc Natl Acad Sci U S A 95:861-868.

Klassen J, Tong C, Flanagan JR (2005) Learning and recall of incremental kinematic and dynamic sensorimotor transformations. Exp Brain Res 164:250-259.

Kluzik J, Diedrichsen J, Shadmehr R, Bastian AJ (2008) Reach adaptation: what determines whether we learn an internal model of the tool or adapt the model of our arm? J Neurophysiol 100:1455-1464.

Kruskal WH, Wallis WA (1952) Use of ranks in one-criterion variance analysis. J Am Stat Assoc 47:583-621.

Malfait N, Ostry DJ (2004) Is interlimb transfer of force-field adaptation a cognitive response to the sudden introduction of load? J Neurosci 24:8084-8089.

Mazzoni P, Krakauer JW (2006) An implicit plan overrides an explicit strategy during visuomotor adaptation. J Neurosci 26:3642-3645.

Medina JF, Garcia KS, Mauk MD (2001) A mechanism for savings in the cerebellum. J Neurosci 21:4081-4089.

Moscovitch C, Kapur S, Köhler S, Houle S (1995) Distinct neural correlates of visual long-term memory for spatial location and object identity: a positron emission tomography study in humans. Proc Natl Acad Sci U S A 92:3721-3725.

Noto CT, Robinson FR (2001) Visual error is the stimulus for saccade gain adaptation. Brain Res Cogn Brain Res 12:301-305.

Rabe K, Livne O, Gizewski ER, Aurich V, Beck A, Timmann D, Donchin O (2009) Adaptation to visuomotor rotation and force field perturbation is correlated to different brain areas in patients with cerebellar degeneration. J Neurophysiol 101:1961-1971.

Redding GM, Wallace B (1996) Adaptive spatial alignment and strategic perceptual-motor control. J Exp Psychol Hum Percept Perform 22:379-394.

Shadmehr R, Mussa-Ivaldi FA (1994) Adaptive representation of dynamics during learning of a motor task. J Neurosci 14:3208-3224.

Shadmehr R, Brandt J, Corkin S (1998) Time-dependent motor memory processes in amnesic subjects. J Neurophysiol 80:1590-1597.

Shallice T, Fletcher P, Frith CD, Grasby P, Frackowiak RSJ, Dolan RJ (1994) Brain regions associated with acquisition and retrieval of verbal episodic memory. Nature 368:633-635.

Sing GC, Smith MA (2010) Reduction in learning rates associated with anterograde interference results from interactions between different timescales in motor adaptation. PLoS Comput Biol 6:e1000893.

Smith EE, Jonides J, Koeppe RA (1996) Dissociating verbal and spatial working memory using pet. Cereb Cortex 6:11-20.

Smith MA, Shadmehr R (2005) Intact ability to learn internal models of arm dynamics in Huntington's disease but not cerebellar degeneration. J Neurophysiol 93:2809-2821.

Smith MA, Ghazizadeh A, Shadmehr R (2006) Interacting adaptive processes with different timescales underlie short-term motor learning. PLoS Biology 4:e179.

Squire LR (1992) Declarative and nondeclarative memory: multiple brain systems supporting learning and memory. J Cogn Neurosci 4:232-243.

Taylor JA, Thoroughman KA (2007) Divided attention impairs human motor adaptation but not feedback control. J Neurophysiol 98:317-326.

Taylor JA, Thoroughman KA (2008) Motor adaptation scaled by the difficulty of a secondary cognitive task. PloS One 3:e2485-e2485.

Tseng YW, Diedrichsen J, Krakauer JW, Shadmehr R, Bastian AJ (2007) Sensory prediction errors drive cerebellum-dependent adaptation of reaching. J Neurophysiol 98:54-62.

Wallman J, Fuchs AF (1998) Saccadic gain modification: visual error drives motor adaptation. J Neurophysiol 80:2405-2416. 